\title{
Atravessamentos culturais de gênero e educação: discursos de preconceito implícito
}

\section{de alunos da saúde}

\author{
Cultural crossings of gender and education: discourses of implicit prejudice of health students \\ Cruces culturales de género y educación: discursos de prejuicio implícito de los estudiantes de salud
}

Recebido: 24/11/2021 | Revisado: 02/12/2021 | Aceito: 03/12/2021 | Publicado: 13/12/2021

Vera Elenei da Costa Somavilla

ORCID: https://orcid.org/0000-0001-9759-8659

Universidade de Santa Cruz do Sul, Brasil

E-mail: veras@unisc.br

Analídia Rodolpho Petry

ORCID: https://orcid.org/0000-0002-8995-0138

Universidade de Santa Cruz do Sul, Brasil

E-mail: analidiapetry@gmail.com

Marina Horn

ORCID: https://orcid.org/0000-0001-7669-5679

Universidade de Santa Cruz do Sul, Brasil

E-mail: marinahorn781@gmail.com

Iagro Cesar de Almeida

ORCID: https://orcid.org/0000-0003-0697-9910

Universidade de Santa Cruz do Sul, Brasil

E-mail: iagroalmeida@gmail.com

Ana Carolina Bienert

ORCID: https://orcid.org/0000-0003-1057-5345

Universidade de Santa Cruz do Sul, Brasil

E-mail: anabienert23@gmail.com

Guilherme Mocelin

ORCID: https://orcid.org/0000-0001-9727-3619

Universidade de Santa Cruz do Sul, Brasil

E-mail: mocelinguilherme@gmail.com

\begin{abstract}
Resumo
Esse trabalho tem por objetivo investigar a produção de preconceito implícito nos discursos de alunos de graduação da área da saúde e seus atravessamentos culturais com gênero e educação. Quanto a metodologia utilizada, trata-se de um estudo qualitativo descritivo, desenvolvido com estudantes de cursos da área da saúde de uma universidade brasileira e uma espanhola. A coleta de dados deu-se a partir de entrevistas semiestruturadas gravadas em áudios e transcritas. Para a análise de dados, fez-se uso da técnica de Análise de Conteúdo. Dessa forma, as questões culturais e educacionais observadas nos discursos, levam em consideração os aspectos (des)construídos e gerados ao decorrer da vida; os quais convidam a refletir acerca dos modelos sociais, considerando questões heteronormativas e modelos unidirecionais. Marcas que reforçam o preconceito implícito enraizados na sociedade civil, bem como os discursos que trazem os preconceitos velados, são responsáveis por enfraquecer um modelo social igualitário e equitativo. Desse modo, constatamos que o estudo provocou e evidenciou a necessidade de reflexões sobre o processo formativo dos acadêmicos da saúde.
\end{abstract}

Palavras-chave: Binarismo de gênero; Educação; Preconceito; Cultura; Saúde pública.

\begin{abstract}
This work aims to investigate the production of prejudice implicit in the speeches of undergraduate students in the health area and its cultural crossings with gender and education. As for the methodology used, it is a descriptive qualitative study, developed with students from courses in the area of health at a Brazilian and a Spanish university. Data collection took place through semi-structured interviews recorded in audio and transcribed. For data analysis, the Content Analysis technique was used. Thus, the cultural and educational issues observed in the speeches take into account the (de)constructed and generated aspects throughout life; which invite you to reflect on social models, considering heteronormative issues and unidirectional models. Brands that reinforce implicit prejudice rooted in civil society, as well as discourses that bring veiled prejudices, are responsible for weakening an egalitarian and equitable social model. Thus, we found that the study provoked and highlighted the need for reflection on the educational process of health academics.
\end{abstract}

Keywords: Gender binarism; Education; Prejudice; Culture; Public health. 


\begin{abstract}
Resumen
Este trabajo tiene como objetivo investigar la producción de prejuicios implícitos en los discursos de estudiantes de pregrado en el área de la salud y sus cruces culturales con el género y la educación. En cuanto a la metodología utilizada, se trata de un estudio cualitativo descriptivo, desarrollado con estudiantes de cursos en el área de la salud en una universidad brasileña y española. La recolección de datos se realizó a través de entrevistas semiestructuradas grabadas en audio y transcritas. Para el análisis de los datos se utilizó la técnica de Análisis de Contenido. Así, las cuestiones culturales y educativas observadas en los discursos toman en cuenta los aspectos (des) construidos y generados a lo largo de la vida; que invitan a reflexionar sobre modelos sociales, considerando temas heteronormativos y modelos unidireccionales. Las marcas que refuerzan los prejuicios implícitos arraigados en la sociedad civil, así como los discursos que traen prejuicios velados, son responsables de debilitar un modelo social igualitario y equitativo. Así, encontramos que el estudio provocó y destacó la necesidad de una reflexión sobre el proceso educativo de los académicos de la salud.
\end{abstract}

Palabras clave: Binarismo de género; Educación; Perjudicar; Cultura; Salud pública.

\title{
1. Introdução
}

O processo de construção de gênero não ocorre da mesma maneira que o sexo natural e dedutivo, pois esse constituise a partir de uma visão interpretativa das possibilidades de existência, em síntese, a definição de gênero caracteriza-se como um efeito e uma mediação cultural (Oliveira, 2020). A formação e compreensão acerca de gênero e sexualidade acontece por meio de diversas articulações teóricas e práticas. Essa edificação apresenta-se nas mais distintas situações, elabora-se explicitamente, ou não, por um infindável conjunto de súplicas culturais e sociais, configurando este processo como minucioso, delicado e em constante (des)construção. Nesse contexto, família, escola, religião, instituições legais e médicas configuram-se como instâncias relevantes para esse desenvolvimento, uma vez que suas concepções se apresentaram, durante muito tempo, como absolutas e quase soberanas (Foucault, 2019).

Segundo Homem e Calligaris (2019), a sociedade humana elabora diversos conceitos, conscientemente ou não. Dessa forma, as ideias que se apresentam mais naturalmente, tendo como exemplo as oposições: masculino/feminino, pobres/ricos ou selvagens/civilizados, na verdade foram construídas. E, por sinal, só é possível percebê-las singularmente a partir do estágio em que nos posicionamos questionando ou (des)construindo tais conceituações. Todavia, provavelmente, essas ideias são úteis para a constituição do sujeito, não permitindo o seu descarte do cenário cultural por completo. O revés seria tomar estas representações binárias como definitivas, uma vez que a arquitetura humana se dá sempre fluída e nunca estamos construídos por completo. Portanto, sendo pensante e criativa, a humanidade pode, felizmente, modificar-se a qualquer momento sua forma de enxergar a si e o mundo (Mendes et al., 2016).

Experimenta-se um contexto político hodierno, onde a intolerância prevalece nos discursos dos grandes líderes mundiais, apesar de muito se falar em educar para a diferença. Essas falas intransigentes acabam por acarretar ainda mais em negacionismo em torno de pautas importantes e extremamente necessárias como as discussões de gênero diante dos espaços educadores/formadores. A pesquisadora brasileira Guacira Lopes Louro, em sua obra "Gênero, sexualidade e educação: uma perspectiva pós-estruturalista (1997)", que foi um marco nessa temática, passa a nos trazer questões sobre o estudo de gênero e educação e, acaba por romper referências biologizantes antes predominantes. Partindo do pressuposto de que para instruir sobre a sexualidade, precisamos preparar os educadores, é imprescindível discutirmos o papel fundamental que a linguagem apresenta como agente de censura e preconceito implícito perante a sociedade.

O termo viés implícito ou preconceito implícito está relacionado a forma com que nos comportamos e agimos inconscientemente frente a uma situação ou grupo social. Essa expressão recebeu maior destaque em 1998, quando Banaji e Greenwald publicaram o Teste de Associação Implícita (IAT). Posteriormente, o teste foi aplicado em outros ensaios e demonstrou sua eficácia em comprovar o tal viés e seu impacto. No que se refere a temática desse estudo e também podemos citar que atualmente, com o início da pandemia do novo Coronavírus, pesquisas indicaram a manifestação de outras formas de repúdio, como por exemplo, violência e discriminação contra os profissionais de saúde (Aydogdu, 2020). 
Os modelos que norteiam as reflexões em relação à sociedade, em sua grande maioria compreendem que os seres humanos são motivados para satisfazer suas necessidades humanas básicas, fisiológicas, de segurança, social, de autoestima e de realizações pessoais. As relações sociais estão articuladas com as relações de consumo comprovando que o comportamento das pessoas são influenciáveis pelo fato da necessidade de aceitação (França, 2019). No contexto acadêmico a necessidade de aceitação pode ser observada em diversas situações e também pode ser um fator que desencadeia manifestações preconceituosas e discriminatórias, mesmo que o espaço universitário promova discursos de inclusão, democracia e igualdade. Assim sendo, o presente estudo traz como objetivo: investigar a produção de preconceito implícito nos discursos de alunos de graduação da área da saúde e seus atravessamentos culturais com gênero e educação.

\section{Metodologia}

Trata-se de uma pesquisa qualitativa exploratória descritiva (Lacerda et al., 2018) que se origina de um estudo maior cognominado Gênero e Biotecnologias - Interfaces entre discursos e instituições na formação de alunos dos cursos da área da saúde. Como local do estudo optou-se por realizar em uma universidade brasileira localizada na região centro-leste do estado do Rio Grande do Sul e em uma universidade localizada na região da Catalunha, Espanha. Os sujeitos que compuseram a amostra formaram um grupo constituído por alunos de graduação da área da saúde da Universidade de Santa Cruz do Sul (Brasil) e da Universidade de Rovira I Virgili (Espanha), que cursavam, a partir do terceiro semestre de cursos da saúde no momento da coleta de dados, que se deu no ano de 2018, independente de suas idades.

Indo ao encontro dos objetivos do presente estudo, como método de coleta de dados, optou-se pela realização de entrevistas semiestruturadas, fazendo o uso de gravador em áudio para produção de todos os registros verbais expressos durante a entrevista. Posterior a isso, as falas foram transcritas fidedignamente, transformando-as em textos, de modo a facilitar a interpretação e releitura por parte dos pesquisadores. Para o mantimento do anonimato dos sujeitos faz-se uso de codinomes, sendo eles as iniciais do seu curso, seguido da numeração arábica que descreveu a ordem de acontecimentos das entrevistas (Enfermagem=E; Medicina=M, Fisioterapia=F, acrescido dos números 1; 2; 3 e assim por diante).

Para contemplar integralmente a proposta de análise dos dados, fez-se uso da técnica de Análise de Conteúdo com a finalidade de verificar as proposições dos discursos do campo dos estudos culturais que se alinharam por afinidades das respostas. Tal técnica é composta por classificação, pré-análise e análise, na qual utilizou-se para verificar os dados obtidos de forma que ficasse organizada as informações, determinando o que é importante e o que ainda falta pesquisar. Posteriormente, com base na etapa já descrita, realizou-se a exploração do material, que consiste em estruturar os achados com o propósito de analisar e formar as categorias temáticas analíticas, realizando a consolidação, sistematização e tratamento com base no que foi explicitado (Minayo, 2014; Bardin, 2011).

Sob esse prisma e em atendimentos a resolução do Conselho Nacional de Saúde no 466/12 o presente estudo foi submetido e aprovado pelo Comitê de Ética em pesquisa da UNISC sob o parecer ético nº 3.327.608.

\section{Resultados}

Nos resultados que seguem serão observados os achados do estudo, divididos em dois grupos, de modo a dinamizar a compreensão. No primeiro momento serão abordadas as questões culturais e educacionais observadas nos discursos de acadêmicos da área da saúde, considerando os valores (des)construídos e os aspectos gerados durante o curso da vida: Cultura e educação: questões de corpo, gênero e sexualidade no ensino superior. Em segundo momento hão de ser explicitados os discursos de participantes da pesquisa pontuando as marcas que reforçam os preconceitos implícitos: Preconceito Implícito: discursos de discentes. 
Cabe ressaltar que ambos os assuntos tangenciam as condições existenciais de sociedade e formação de sujeitos, bem como, das relações que se estabelecem e emergem entre profissionais e indivíduos que se encontram em um mesmo cenário. Tal cenário, pode ser decorrente das condições de atuação profissional e utilização de um serviço de saúde, como pode ser notado nos discursos que seguem:

\section{Cultura e educação: questões de corpo, gênero e sexualidade no ensino superior}

Os discursos que marcam a presente abordagem acerca da temática de cultura e educação convidam-nos a refletir acerca de modelos sociais construídos ao longo do curso humano, considerando os aspectos heteronormativos e os modelos unidirecionais de pensamentos. A partir disso, o Quadro 1 sintetiza as categorias e trechos das entrevistas realizadas acerca do tema Cultura e Educação.

Quadro 1 - Discursos de acadêmicos da área da saúde acerca da temática Cultura e Educação.

\begin{tabular}{|c|c|}
\hline CATEGORIAS & TRECHOS DAS ENTREVISTAS \\
\hline $\begin{array}{l}\text { Cultura e } \\
\text { Educação }\end{array}$ & 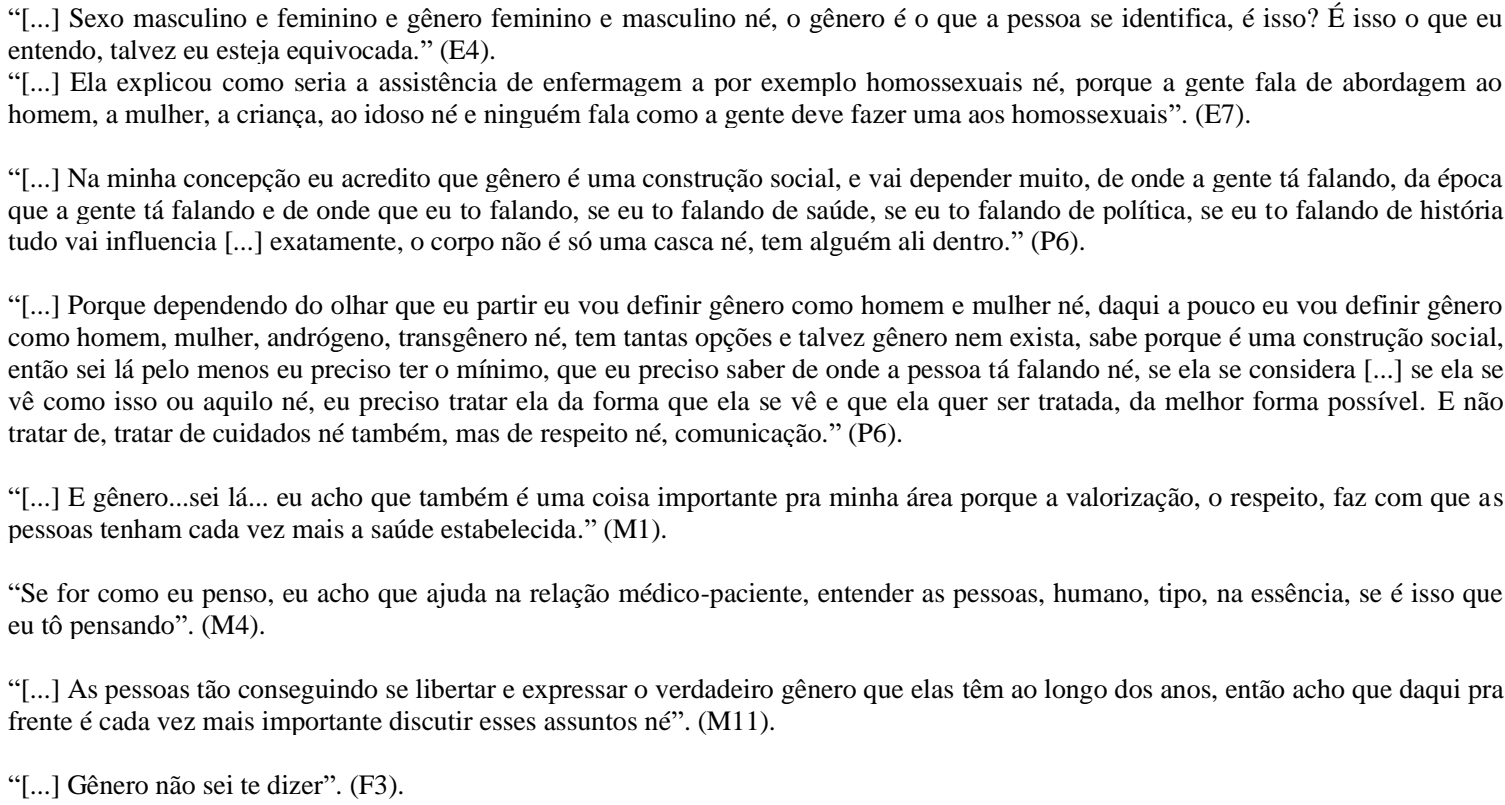 \\
\hline
\end{tabular}

Fonte: Banco de dados da pesquisa (2018).

Como pode se perceber nas falas dos participantes intitulados E4 e E7 (no quadro 1), onde denota-se esse modelo de educação e forma de pensar, tensionam-se as questões e maneiras de observar o layout como a sociedade se expressa e vive. Entender a vida e as relações de uma maneira, não significa ela - a compreensão, a vida, as relações - ser a única forma existente, tampouco uma verdade.

A cultura, o modelo sócio/educacional do estado e família e as relações, exercem forte e constante influência das lentes pelas quais observa-se o todo que nos cerca. A forma como buscam-se os modelos de educação, resulta em importantes fontes e paradigmas de expressões para os atendimentos futuros desses profissionais da saúde em formação. Equidade e humanização são pressupostos que deveriam atravessar a maneira como são realizados os contatos humanos, considerando a empatia e singularidade dos sujeitos nessa tríade: profissional, usuário e educação/cultura.

No tocante dessa temática, fora evidenciado discursos que percebem a vasta forma de expressão que os indivíduos em sociedades são capazes de assumir, ou mesmo em não assumir, compreendendo que os fatores que visam dividir/discriminar em "caixas/categorias" ou mesmo encaixotar, ao mesmo tempo, por vezes, sem a ciência, culmina no enfraquecimento das 
lutas de classes e grupos sociais por espaços e reconhecimentos humanos básicos, como o direito à vida. O participante P6 do quadro 1, convida-nos e submergir no complexo e vasto universo das maneiras como as pessoas se veem e enxergam o mundo, entendendo que a "casca do corpo" embora seja um cartão de visita à sociedade hodierna, não é estático e não configura uma forma que deva permanecer imutável se assim for do desejo desse sujeito.

Sob a outra face desse prisma observam-se os discursos dos sujeitos M1, M4, M11 e F3, ainda referente ao Quadro 1, os quais reproduzem representações que desvelam modelos educacionais enfraquecidos e limitados, tanto a nível cultural como educacional básico e superior. Por meio das expressões captadas no momento da produção dos dados e nos discursos, torna-se sugestivo que a abordagem da temática LGBTQIA+, para estes, é justa pois a comunidade científica está abordando acerca de, e isso lhes deixará "a luz" de espaços que proporcionam visibilidade profissional e social por interesses - quiçá econômicos. Somados, talvez a protestos valorativos pessoais individualizados que não buscam ou visam a diminuição das desigualdades entre os sujeitos, mas sim, por prestígio e, quem sabe fatias de mercado.

\section{Preconceito implícito: discursos de discentes}

Como resultante da pesquisa realizada com base na entrevista de acadêmicos da área da saúde, emergiu a categoria que se refere ao preconceito implícito no cotidiano desses alunos. Estas temáticas podem ser observadas de diversas maneiras, como podemos observar nos trechos abaixo do Quadro 2:

Quadro 2 - Discursos de acadêmicos da área da saúde acerca da temática preconceito implícito.

\begin{tabular}{|c|c|}
\hline CATEGORIAS & TRECHOS DAS ENTREVISTAS \\
\hline $\begin{array}{l}\text { Preconceito } \\
\text { Implícito }\end{array}$ & 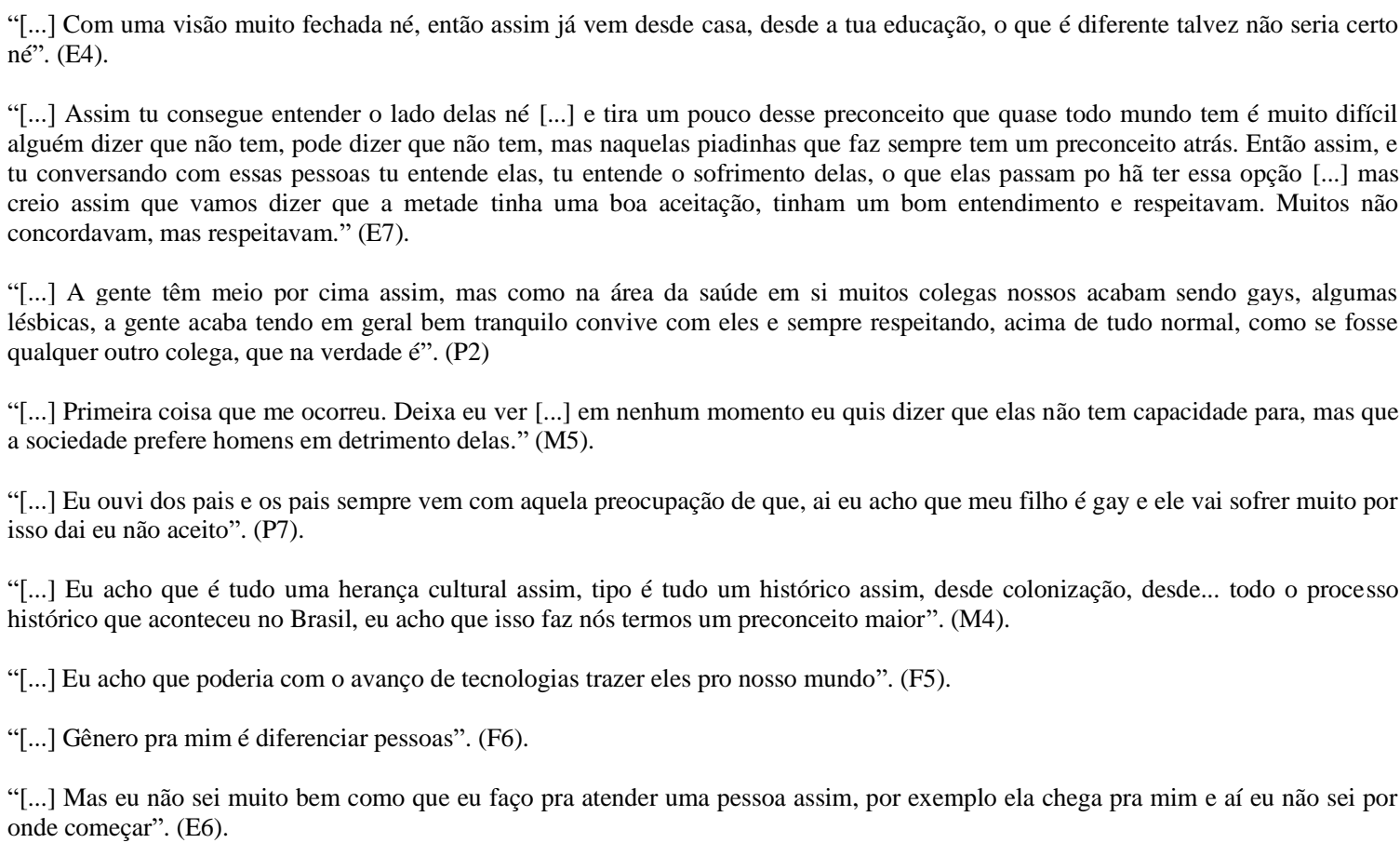 \\
\hline
\end{tabular}

Fonte: Banco de dados da pesquisa.

Na categoria Preconceito Implícito, podemos observar como está enraizado em nossa sociedade determinados tabus, onde o entrevistado $\mathrm{E} 4$ diz que "O que é diferente talvez não seria certo, né", reforçado discursos que parecem estar enraizados na sociedade. Observa-se que as falas expressam de distintas formas o quanto gênero está vinculado a uma multiplicidade de preconceitos, que parecem estar internalizados e, em seus discursos os sujeitos não percebem o quanto esta é 
uma postura defensiva para esse assunto que relaciona a escolha da sexualidade. Esse aspecto é reforçado na fala de F5 "Trazer eles para o nosso mundo". Como se as diferenças levassem à comunidade LGBTQIA+ a não compor o mundo convencionalmente hegemônico.

Em contrapartida, temos uma fala importante, trazendo outro ponto de vista, de forma a transparecer um pensamento mais aberto quanto ao assunto. E diferente dos demais entrevistados, ele discorre que: "Tentar entender como que o paciente se sente ou se expressa naquela condição dele, eu acho que isso facilita até na verdade no tratamento que tu vai fornecer para ele" (M11). Conduzindo-nos a (des)construir modelos de pensamentos que destoam das formas por vezes, agressivas, relacionadas a discursos que trazem o preconceito velado, responsável por segmentar e enfraquecer um modelo de sociedade igualitária e equitativa à luz dos conceitos de vida livre, com liberdades de expressão em todas as formas.

\section{Discussão}

Para discutirmos as noções de corpo, gênero e sexualidade na cultura e na educação, tornou-se necessário a retomada de alguns autores importantes, que explicam a "educação bancária" como o ato de depositar. Para ele, este ato caracteriza o educador como depositante do saber sobre os depositários, os educandos. Neste caso, a relação que fora estabelecida a partir do processo de educação, é a de transferência de valores e conhecimentos. Desta forma, se identificou que o formato institucional de aprendizado não se dá de maneira neutra, geralmente se assemelha aos interesses da classe dominante e, consequentemente, as abordagens dos conteúdos ensinados tendem a seguir os seus ideais e as lógicas capitalistas de pensar (Almeida, 2021; Freire, 1987).

No Brasil, diferente do neoliberalismo norte-americano, o estado se torna um ator central. E neste âmbito, atribui-se também a ele, a responsabilidade legal para com a saúde geral da população brasileira, conforme apresenta-se no artigo $3^{\circ}$, inciso IV, da Constituição Federativa (1988), (Bento, 2017; Brasil, 1988). Com isso, entre os brasileiros, o debate sobre a saúde da população LGBTQIA+ também se aloja nestes preceitos que circundam acerca da saúde/estado/cidadania. Nesse momento, foi possível identificar um "nó político" que se estabelece de forma peculiar, pois, se nos Estados Unidos o atendimento de saúde para pessoas trans, bi, gays, entre outros, se aproxima de uma teatralização, porém assumida como necessária, e na realidade do Brasil não seria necessário reproduzir esse cuidado como um jogo de cena, questiona-se qual seria o sentido de lançar mão de um acolhimento, ou atendimento, qualificado para pessoas LGBTQIA+, seguindo o que o SUS nos explicita de forma clara em sua redação.

Reforçando o papel do Estado no cuidado de saúde, no Brasil temos a Política Nacional de Humanização (PNH) do SUS, onde são estabelecidos princípios para o cuidado da população, que se baseiam no reconhecimento das demandas do usuário, a fim de legitimar sua necessidade de saúde. Um dos principais princípios se refere ao acolhimento. O acolher deve seguir e sustentar a relação entre profissional/usuário e equipe/serviço, portanto este constitui-se de forma coletiva e seu objetivo é fortalecer as relações, vínculos e compromissos, criando uma relação socioafetiva (Brasil, 1988). Corroborando com isto, no artigo $196^{\circ}$ da Constituição Federativa de 1988, obtém-se a seguinte afirmação, que se aproxima das ideias citadas anteriormente:

"A saúde é direito de todos e dever do Estado, garantido mediante políticas sociais e econômicas que visem à redução do risco de doença e de outros agravos e ao acesso universal e igualitário às ações e serviços para sua promoção, proteção e recuperação" (Constituição Federativa, 1988).

$\mathrm{Na}$ afirmativa supracitada, constatou-se que o direito à saúde e aos serviços essenciais são de todos. Esse "todos" refere-se integralmente à população do país, evitando distinções entre cor, raça, idade, gênero, reforçando o que está escrito no artigo $5^{\circ}$ da mesma constituição: "Todos são iguais perante a lei” (Brasil, 1988, p. 118).

Indo ao encontro desta proposição das políticas públicas de saúde e aqui especialmente a PNH, foi possível 
reconhecer a existência de materiais e métodos para nortear a abordagem mais adequada aos usuários, independentemente de serem pertencentes à população LGBTQIA+. Porém, observou-se a fala: "Ela explicou como seria a assistência de enfermagem a por exemplo homossexual né, porque a gente fala de abordagem ao homem, a mulher, a criança, ao idoso né e ninguém fala como a gente deve fazer uma aos homossexuais" (E7).

Identificou-se, através da diferenciação da categoria criada para homossexuais, uma necessidade, por parte do(a) estudante, de uma "abordagem", que por sua vez correlaciona-se com o acolhimento, diferenciada para tal população. Homossexuais, antes de pertencerem a este marcador social, podem possuir uma identidade de gênero, isto faz com que não se torne necessário elencar mais uma categoria para englobar esta população. Uma vez que, o "ser homossexual" apenas denota a orientação sexual do paciente e as noções de alteridade quanto as sexualidades têm sua "origem" no tecido social, pois biologicamente, o corpo do sujeito hetero ou homossexual apresentou-se com as mesmas características anatômicas (Marmelstein, 2021).

Outrossim, ao versar sobre a temática que aborda acerca do preconceito implícito, tornou-se evidente, modelos e forma de agir de maneira sutil diante dos sujeitos e para com os sujeitos, ou seja, um manifesto brando, inconsciente e até mesmo involuntário que se expressa de forma agressiva e depreciativa com as quebras de padrões de grupos humanos. Tais aspectos podem ser resultantes de fatores culturais e educativos que seguem os sujeitos e os moldam enquanto sociedade (Souza et al., 2020; Alves et al., 2020). O preconceito implícito é, de fato, um gerador de diversos comportamentos, práticas e crenças que fundamentam desigualdades confutáveis e improcedentes, baseadas em um sistema cultural.

A heteronormatividade por vezes corrobora com modelos agressivos de expressões, que são incorporadas pela sociedade e produzem impactos nas relações sociais. Por estarem fortemente arraigado nas estruturas formadoras, essa cultura (por vezes preconceituosas) carece de largas discussões e a fim de ser entendida como constitutiva de um modelo opressor, que pode resultar em danos aqueles e aquelas que são prejudicados por incapacidade de existir da forma que se sentem felizes (Pires et al., 2019).

Os discursos que expressam as distintas formas de preconceito atravessam e são atravessados pelas idas e vindas de uma evolução. Partindo do pressuposto de que as formas que conhecemos sobre preconceito são construções sociais, que materializam distintas formas de intolerância, que afetam aqueles que a praticam e aqueles que sofrem. É comum os sujeitos observarem o que foge de padrões de maneira diferente, todavia, não se justifica a marginalização e exclusão destes grupos, por pensar, agir e sentir, fora de padrões que a própria sociedade ergueu em torno de si (Lacerda; Couto; Nogueról, 2020; Moretti-Pires et al, 2019).

\section{Considerações Finais}

A pesquisa da qual deriva a escrita deste artigo, parte de pressupostos de vários encontros, quais sejam; o encontro de duas instituições de Ensino de países distintos (Brasil e Espanha), o encontro de cursos da área da saúde - enfermagem, psicologia, medicina, fisioterapia, odontologia e farmácia -, e o encontro com a temática "gênero". São encontros potentes na medida que oferecem possibilidades de reflexão, trazidas pelas falas dos estudantes que participaram desta pesquisa, e que produzem uma série de indicativos relacionados à temática de gênero e à graduação na área da saúde.

As análises desenvolvidas pretendem fazer sentido, para povoar pensamentos, e possibilidades no agir desses futuros profissionais nos seus encontros com a população LGBTQI+. As categorias analíticas estiveram centradas no que se refere aos aspectos culturais e ao preconceito implícito.

No que se refere à análise sobre os aspectos culturais, os estudantes entrevistados destacam, que estes corroboram para que as normas hegemônicas relacionadas a heteronormatividade se reproduzam tanto na formação, quanto na assistência à saúde. Os dados pontuam que os usuários são classificados em "caixas" o que de certo modo, limita a percepção das 
individualidades, em especial relacionadas à sexualidade. Indicando assim a urgência de ações metodológicas que possam embaralhar, tensionar, reconfigurar normas culturais, tomadas como "verdades" no cuidado à saúde. Contemplando a construção de habilidades para que os universitários e futuros profissionais possam se sentir mobilizados e envolvidos em ações e experiências onde as escolhas sexuais não sejam um fator que possa gerar descriminação e preconceito.

Por sua vez, aspectos de preconceito implícito emergem das análises como um modelo internalizado, pouco reconhecido como forma de preconceito. Entretanto, são trazidos e tidos como normas já construídas e difíceis de tomarem novos rumos, conforme os participantes da pesquisa. As ações que envolvem atitudes preconceituosas constroem e potencializam grandes dimensões, que se tornam potentes em seus impactos negativos e reproduzem sofrimento, discriminação e segmentação de sociedade.

Deste modo a análise, pretende provocar reflexões sobre os processos formativos e a importância da temática gênero na graduação em saúde. Vislumbrando possibilidades que favoreçam a potência do agir dos futuros profissionais no que se refere a qualificação do cuidado da população LGBTQI+. Para que isso seja possível é necessário além de provocar reflexões, acreditar na potencialidade de que a abordagem de gênero é um tema de extrema importância no currículo para que possamos estar mais atentos na relação nos encontros que nos tocam e que produzem saúde. Ainda, sugerimos que novos estudos venham a abordar a presente temática para que a visibilidade posso ser potencializada e discursos de preconceitos minimizados.

\section{Referências}

Almeida, L. C. (2021) Paulo Freire: present! Bibliographic survey in Educação \& Sociedade. Rev Educ Soc, 42(e254030), 1-24.

Alves, C. N., Wilhelm, L. A., Prates, L. A., Silva, S. C. da, Tronco, C. S., Cremonese, L., \& Sehnem, G. D. (2020). Práticas de cuidado realizadas por enfermeiras durante o pré-natal de baixo risco: bases para o cuidado cultural. Research, Society and Development, 9 (7), e999975275.

Aydogdu, A. L. F. (2020) Violence and discrimination against healthcare workers in times of new coronavírus. Journal of Nursing and Health. Universidade Federal de Pelotas.

Bardin, Laurence (2011). Análise de Conteúdo. (4a ed.), Edições 70.

Bento, B. (2017). Transviad@s: gênero, sexualidade e direitos humanos. EDUFBA.

Brasil (2013). Política Nacional de Humanização - $\quad P N H . \quad 1^{\text {a }}$ edição. Brasília, Ministério da Saúde. https://bvsms.saude.gov.br/bvs/publicacoes/politica_nacional_humanizacao_pnh_folheto.pdf.

Brasil (2016). Constituição da República Federativa do Brasil: texto constitucional promulgado em 5 de outubro de 1988 , com as alterações determinadas pelas Emendas Constitucionais de Revisão nos 1 a 6/94, pelas Emendas Constitucionais nos 1/92 a 91/2016 e pelo Decreto Legislativo no 186/2008. Brasília, 2016. https://www2.senado.leg.br/bdsf/bitstream/handle/id/518231/CF88.

Foucault, M. (2019). Reflexões acerca dos saberes e dos sujeitos. Edições EdUERN.

França, S. (2019). Pirâmide de Maslow: Como impacta no desenvolvimento pessoal. https://www.slacoaching.com.br/artigos-do-presidente/piramide-demaslow-como-impacta-no-desenvolvimento-pessoal.

Freire, P. (1987). Pedagogia do Oprimido. (17a ed.), Paz e Terra.

Greenwald, A. G., McGhee, D. E., \& Schwartz, J. L. K. (1998). Measuring individual differences in implicit cognition: The implicit association test., Journal of Personality and Social Psychology. American Psychological Association (APA) 74, p. 1464-80.

Herculine, B. (1982). O diário de um hermafrodita. Edições 70.

Homem, M., \& Calligari, C. (2019). Coisa de menina? Uma conversa sobre gênero, sexualidade, maternidade e feminismo. (3a ed.), Papirus 7 mares.

Lacerda, A. C., Couto, J. M., \& Nogueról, L. P. F. Celso Furtado em seu centenário de nascimento: pensamento e ação. Rev Estud Av, 34 (100), 291-304

Lacerda, M. R., Ribeiro, R. P., \& Costenaro, R. G. S. (2018). Metodologias da pesquisa para a enfermagem e saúde: da teoria à prática. $2^{\mathrm{a}}$ ed. Porto Alegre: Moriá Editora.

Louro, G.L. (1977). Gênero, sexualidade e educação: Uma perspectiva pós-estruturalista. (6a ed.), Vozes.

Marmelstein, G. (2021). Discriminação por preconceito implícito. Editora Juspodivm.

Mendes, R., Pezzato, L.M., \& Sacardo, D.P. (2016). Pesquisa-intervenção em promoção da saúde: desafios metodológicos de pesquisar “com”. Ciência \& Saúde Coletiva, 21, 1737-46. 
Research, Society and Development, v. 10, n. 16, e350101623875, 2021

(CC BY 4.0) | ISSN 2525-3409 | DOI: http://dx.doi.org/10.33448/rsd-v10i16.23875

Minayo, M. C. S. (2014). O desafio do conhecimento: pesquisa qualitativa em saúde. (14a ed.), HUCITEC.

Moretti-Pires, R. O., Guadagnin, L. I., Tesser-Júnior, Z. C., Campos, D. A., \& Turatti, B. O. (2016). Preconceito contra Diversidade Sexual e de Gênero entre

Estudantes de Medicina de $1^{\circ}$ ao $8^{\circ}$ Semestre de um Curso da Região Sul do Brasil, Revista Brasileira de Educação Médica, 24(1), 557-67.

Oliveira A. (2020). Ficções porno-políticas do corpo (a partir) de Preciado, Revista Estudos Feministas. FapUNIFESP, 28(5), 1-13.

Souza, A. B., Alves, G. D., Silveira, L. A., Oliveira, L. C., Lazzaretti, L. N, Battisti, S. C., et al (2020). Os impactos do preconceito social e familiar na saúde mental das lésbicas, gays, bissexuais e transsexuais Research, Society and Development, 9(2), e34942760. 